\title{
Renal Allograft Thrombosis
}

National Cancer Institute

\section{Source}

National Cancer Institute. Renal Allograft Thrombosis. NCI Thesaurus. Code C114844.

Thrombus formation within the arterial or venous system of a donor kidney post transplantation. 